\title{
Biochemia Medica's editorial policy on authorship
}

\author{
Vesna Supak-Smolcic*1,2,3, Ana-Maria Simundic1,4 \\ ${ }^{1}$ Biochemia Medica, Zagreb, Croatia \\ ${ }^{2}$ Clinical Department for Laboratory Diagnostics, Clinical Hospital Centre Rijeka, Rijeka, Croatia \\ ${ }^{3}$ Department of Medical Informatics, Rijeka University School of Medicine, Rijeka, Croatia \\ ${ }^{4}$ University Department of Chemistry, University Hospital Centre Sestre milosrdnice, Zagreb, Croatia \\ *Corresponding author: vesnasupak@gmail.com
}

\begin{abstract}
Recent findings of authorship criteria violations in the manuscripts submitted to Biochemia Medica show that almost $40 \%$ of authors do not meet necessary criteria for authorship and thus indicate the need for better dissemination of editorial policy on authorship in our journal. We believe that such cases are mostly due to the authors' unawareness or the lack of understanding of the authorship criteria. With this article we therefore wish to declare our editorial policy on authorship and authorship criteria. Biochemia Medica endorses the authorship policy provided by the International Committee of Medical Journal Editors (ICMJE). Information on authorship is assessed by self-reported authorship claims during on-line manuscript submission. Those who meet ICMJE criteria shall be listed as authors, and all listed authors shall fulfill ICMJE criteria. All authors should be responsible for content of the article and have to know other authors' contributions to the study. Biochemia Medica will follow recommendations provided by Committee on Publication Ethics (COPE) flowcharts for possible disputes. By adhering to this procedure we hope to raise awareness about the importance of compliance with ICMJE authorship recommendations.
\end{abstract}

Key words: authorship; contribution; editorial policy; publication; research integrity

Accepted: July 27, 2015

\section{Introduction}

Our recent study based on self-reported contributions by authors of the manuscripts submitted to Biochemia Medica, has revealed that almost $40 \%$ of authors were not eligible for authorship (1). This obviously points to their lack of understanding of the type of contribution that justifies for authorship. Based on those findings it became clear that being an author is a desirable role and that the benefits of authorship are well known among scientists. Credits merited by enlarging the number of co-authored articles such as financial and academic advancement are well described in the literature (2). Authorship can be seen a mean of professional advancement and as such is subjected to manipulation (3). However, benefits come with the responsibility and accountability for all aspects of co-authored work which are compromised every time an author falsely declares his contributions.
To define the role of an author and to prevent possible authorship disputes, International Committee of Medical Journal Editors (ICMJE) gave a set of criteria for authorship and explained the difference between authors and contributors (4). Well established journals are now increasingly adopting the existing international recommendations for authorship criteria and including them in their Instructions for authors. However, policy to define authorship is still quite uncommon in some small society based journals from small scientific communities. One recent study has demonstrated that only $14 \%$ (28/197) of Croatian open access journals address authorship criteria in their Instructions for authors (5).

Even though authorship criteria exist, authorship violations still occur. Since there is no objective 
mean for assessing the truth of self-reported authorship claims, authors' disputes are not uncommon $(2,6,7)$.

Efforts have been made by the publishing community to minimize questionable authorship practice. For example, Authorder ${ }^{\circledR}$ is a simple Microsoft Office Excel based table which provides assistance in establishing the order of authors according to their contribution (8). To distinguish an author from other authors with the same name or initials, a unique numerical identifier called Open Researcer and Contributor ID (ORCID) has been created (9). ORCID has been recognized by many researchers and publishers as a mean for protecting author's identity.

Many written recommendations have been published in order to help reduce questionable behavior and to educate researchers and scientific community $(4,10-12)$. The aim of this article is to declare the editorial policy on authorship and authorship criteria of Biochemia Medica journal in order to provide assistance and guidance to potential authors and other interested individuals.

\section{Biochemia Medica's recommendations and edi- torial policy on authorship}

Biochemia Medica promotes ethical principles in research and aims to prevent any type of authorship violation and research misconduct. Editorial policy on authorship is stated in the Journal's Instructions for authors available at the Journal's web page. In the text below our editorial policy is declared and discussed in details.

\section{Author vs. contributor}

Biochemia Medica has adopted updated authorship criteria set forth by ICMJE in 2013 (13). According to ICMJE, an author is a person who fulfils all four given criteria:

1. "substantial contributions to conception and design, acquisition of data, or analysis and interpretation of data;

2. drafting the article or revising it critically for important intellectual content;

3. final approval of the version to be published;

4. agreement to be accountable for all aspects of the work in ensuring that questions related to the accuracy or integrity of any part of the work are appropriately investigated and resolved."

All researchers that meet all four criteria have to be stated as authors, and all stated authors have to fulfill all those criteria. Those who wrote the article but are not listed as authors are called ghost-writers, whereas those who are listed as authors, despite their negligible contribution, are called honorary authors (guest authors or gift authors). Various kinds of unethical violations of authorship were recently reviewed by Das N and Das S (14).

All authors have to give their permission to be named because each author must be responsible for content of an article and have to know other authors' contributions to the study. Authorship should be based on mutual confidence and agreement.

According to our editorial policy, all those who do not comply with all four criteria may be considered as contributors and their contribution may be mentioned in Acknowledgement section. Examples of contributions that are not sufficient for authorship are: technical help, writing assistance, language translation service, general support and supervision, financial and material support, providers of patient data or samples etc. Contributors listed in the Acknowledgments section must give their permission to be named and corresponding author is responsible to provide this information during on-line manuscript submission process.

\section{Order of authors on the by-line}

The best timing for determining authors' roles and order on the by-line is before starting the research. Since there are commonly accepted definitions for the contribution of each author on the by-line, it is important to clearly communicate each author's obligations and responsibilities at the very beginning.

First author is the author who has done most of the work and has the greatest contribution to the presented study. First position in the by-line usually implies special status and primary responsibility for the study. First authorship is usually taken into consideration for professional advancement of doctoral researcher. There is growing number of 
articles declaring equal contribution of the first two authors (joint first authors) which must be clearly declared in the published article.

Senior author is usually placed on the last position in the by-line. He is a mentor and guarantor of the study who takes part in all phases of the research and manuscript writing. He has the overall responsibility for all work presented in the article.

Corresponding author is responsible for communication with journal editors and readers. This author handles manuscript submission to the journal and provides all information needed during the submission. It is common for the first or last author to be also the corresponding author.

Middle authors are all those researchers who do not contribute to the article the same way as the first and the last authors do, but certainly fulfill authorship criteria. Their order in the by-line should be according to the level of their contribution to the presented study.

It is most important to point out that all authors, regardless of their position on the by-line, must fulfill all four ICMJE criteria for authorship.

\section{Protection of author's identity}

Biochemia Medica promotes novelties in the area of scientific integrity. Therefore, Journal has recently adopted the system by which each author is

\section{References}

1. Supak-Smolcic V, Mlinaric A, Antoncic D, Horvat M, Omazic J, Simundic AM. ICMJE authorship criteria are not met in a substantial proportion of manuscripts submitted to Biochemia Medica. Biochemia Med (Zagreb) 2015;25:324-34. http://dx.doi.org/10.11613/BM.2015.033.

2. Strange K. Authorship: why not just toss a coin? Am J Physiol Cell Physiol 2008;295:C567-75. http://dx.doi. org/10.1152/ajpcell.00208.2008.

3. Shaw D. The prisoners' dilemmas. EMBO reports 2014;15:635-7.

4. International Committee of Medical Journal Editors. Recommendations for the Conduct, Reporting, Editing, and Publication of Scholarly Work in Medical Journals. Availa-

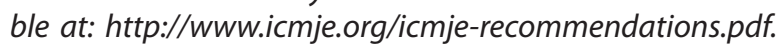
Accessed July 4th 2015. identified with his unique identification number, ORCID. ORCID ensures transparency in authorship and personal identification. Upon registration into the on-line submission system, each potential author is requested to provide his/her ORCID number.

\section{Resolving authorship violations and disputes among authors}

Although Biochemia Medica has adopted ICMJE criteria for authorship, it is the Journal's policy not to further investigate authors' self-reported authorship contributions. We believe that potential authorship criteria violations are mostly due to the authors' unawareness or the lack of understanding of the authorship criteria. We hope that this article will help authors to make easier decisions about authorship in the future.

Should disputes arise among authors of particular manuscript, Biochemia Medica will follow recommendations provided by Committee on Publication Ethics (COPE) flowcharts (15). By respecting this policy and following this procedure we hope to raise awareness among potential authors about the importance of complying with universally accepted recommendations for authorship.

\section{Potential conflict of interest}

None declared.
5. Stojanovski J. Do Croatian open access journals support ethical research? Content analysis of instructions to authors. Biochem Med (Zagreb) 2015;25:12-21. http://dx.doi. org/10.11613/BM.2015.002.

6. Rajasekaran S, Shan RLP, Finnoff JT. Honorary Authorship: Frequency and Associated Factors in Physical Medicine and Rehabilitation Research Articles. Arch Phys Med Rehabil 2014;95:418-28. http://dx.doi.org/10.1016/j. apmr.2013.09.024.

7. Akhabue E, Lautenbach E. "Equal" contribution and credit: an emerging trend in the characterization of authorship. Ann Epidemiol 2010;20:868-71. http://dx.doi.org/10.1016/j. annepidem.2010.08.004.

8. Authorder ${ }^{\circledast}$. Available at: http://www.authorder.com/ home. Accessed July 4th 2015. 
9. ORCID. Available at: http://orcid.org/. Accessed July 4th 2015.

10. Albert T, Wager E. How to handle authorship disputes: a guide for new researchers. The COPE Report 2003;32-4.

11. Vicens $Q$, Bourne PE. Ten simple rules for a successful coIlaboration. PLoS Comput Biol 2007;3:e44. http://dx.doi. org/10.1371/journal.pcbi.0030044.

12. Hess CW, Brückner C, Kaiser T, Mauron A, Wahli W, Wenzel $U J$, et. al. Authorship in scientific publications: analysis and recommendations. Swiss Med Wkly 2015;145:w14108.
13. Simundic AM. News at Biochemia Medica: Research integrity corner, updated Guidelines to authors, revised Author statement form and adopted ICMJE Conflict-of-interest form. Biochem Med (Zagreb) 2013;23:5-6. http://dx.doi. org/10.11613/BM.2013.001.

14. Das N, Das S. Hiring a professional medical writer: is it equivalent to ghostwriting? Biochem Med (Zagreb) 2014;24:1924. http://dx.doi.org/10.11613/BM.2014.004.

15. Committee on Publication Ethics. Flowcharts. Available at: http://publicationethics.org/resources/flowcharts. Accessed July 4th, 2015. 